\title{
The crossover lung segment: congenital malformation associated with a variant of scimitar syndrome
}

\author{
B S CLEMENTS, J O WARNER
}

From the Department of Paediatrics, Cardiothoracic Institute, Brompton Hospital, London

\begin{abstract}
Three cases of a congenitally misplaced segment of lung are described. In one case the abnormal segment was identified at thoracotomy; it extended from an origin in the right upper lobe across the midline into the left hemithorax. The remaining two patients, with identical radiographic features, were identified in a review of 24 cases of bronchovascular malformation. In all three patients the crossover segment was associated with a variant of the scimitar syndrome: right lung hypoplasia with dextrocardia, aberrant systemic arterial supply to the right lower lobe, and anomalous venous drainage of the whole of the right lung, and in two patients with eventration of the right diaphragm.
\end{abstract}

Congenital misplacement of a pulmonary segment in the opposite side of the chest has not previously been described. We present three cases where the radiographic appearances suggest the presence of a crossover segment, which was confirmed at thoracotomy in one case.

\section{Case reports}

CASE 1

A $3.5 \mathrm{~kg}$ girl was delivered of a diabetic mother by caesarian section at 35 weeks' gestation because of evidence of fetal distress. No resuscitation was required but she was noted to be grunting from the age of 2 hours. A chest radiograph showed an opaque right lung field. This was thought to be due to infection with collapse; she was treated accordingly but continued to deteriorate, requiring intubation and ventilation by 10 days of age, at which stage she was transferred to this hospital.

On arrival she was pink, breathing air and had no obvious dysmorphic features, but chest movement and air entry were reduced on the right. Peripheral pulses were all present and the blood pressure was normal in the upper and lower limbs. The cardiac apex was palpable to the right of the sternum. The heart sounds were normal and a grade $2 / 6$ ejection systolic murmur was heard maximally along the right

\footnotetext{
Address for reprint requests: Dr JO Warner, Department of Paediatrics, Cardiothoracic Institute, Brompton Hospital, London SW3 $6 \mathrm{HP}$.
}

Accepted 15 December 1986 parasternal border. The liver and spleen were just palpable. The chest radiograph showed a hypoplastic right lung, which was totally opaque except for a small area in the right lower zone. There was right sided diaphragmatic eventration, and shift of the heart and mediastinum to the right. Right ventricular pressures measured during cardiac catheterisation were three quarters of the systemic pressure. Pulmonary arteriography showed a hypoplastic artery to the right upper lobe with two small branches extending back across the midline posteriorly into the left mid hemithorax (fig 1). The remainder of the hypoplastic right lung was supplied by four aberrant systemic arteries arising from the abdominal and lower thoracic aorta. The venous drainage of the whole of the right lung was anomalous, passing directly to the right atrium (a variant of the scimitar syndrome). The left to right shunt through this anomalous circulation was calculated at 3.7:1. Computed tomography confirmed hypoplasia of the right lung and herniation of the liver through the diaphragmatic eventration, and showed a segment of lung extending from the right upper mid zone across the midline into the left hemithorax posteriorly.

At 4 weeks of age the aberrant systemic arteries were ligated. During the thoracotomy it was noted that the hypoplastic right lung was bilobed, and that a segment of the upper lobe extended across the midline posteriorly behind the dextropositioned heart into the left hemithorax. The whole of the right lung, including the crossover segment, was aerated but the consistency was denser than that of the surrounding normal left lung, in keeping with the appearances seen 


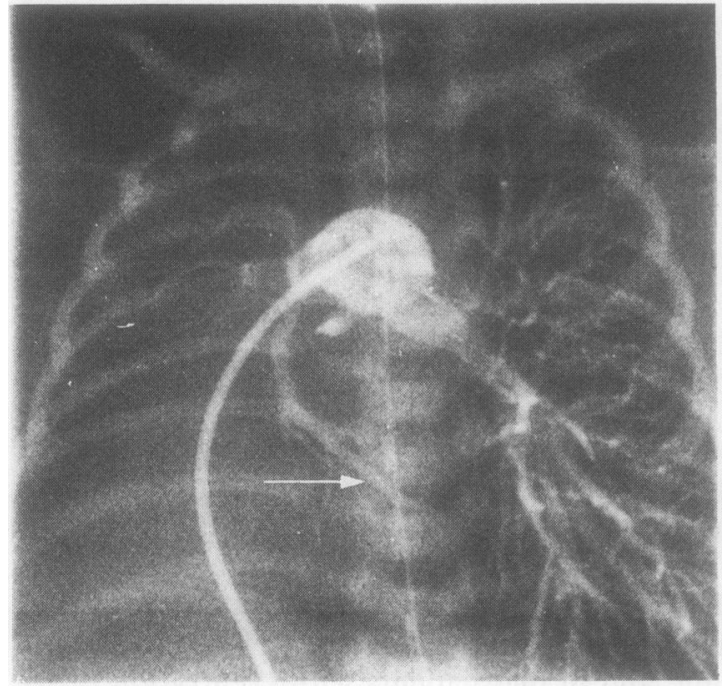

Fig 1 Case 1: Contrast medium injected into the main pulmonary artery outlining a hypoplastic right pulmonary artery with a branch descending medially across the midline into the left hemithorax posteriorly (indicated by arrow).

on the computed tomography scan. The crossover segment retained its bronchial connection with the right upper lobe, and its arterial supply from the hypoplastic right pulmonary artery was confirmed.

Postoperative recovery was prolonged and complicated by recurrent chest infections, one of which proved fatal when the child was 5 months. Permission for necropsy was refused.

\section{CASE 2}

A $2.6 \mathrm{~kg}$ boy was noted to be tachypnoeic with a respiratory rate of 60 breaths a minute at 1 week of age. His apex beat was clearly felt in the fourth right intercostal space. The pulmonary component of the second heart sound was increased and a grade $3 / 6$ ejection systolic murmur was heard along the right sternal edge. The right chest wall was noted to be flatter than the left, with reduced movement and air entry. The chest radiograph showed a hypoplastic right lung with right sided diaphragmatic eventration and dextropositioning of the heart. A clinical diagnosis of the scimitar syndrome was made and the child was referreu to this hospital for further investigation. Cardiac catheterisation confirmed the diagnosis, showing an aberrant systemic arterial supply to the right lower zone and anomalous venous drainage of the whole of the right lung. Injection of contrast into the main pulmonary artery outlined a normal left pulmonary arterial tree and a hypoplastic right pulmonary artery to the right upper lobe. Selective injection into this right pulmonary artery outlined a branch extending back across the midline into the left

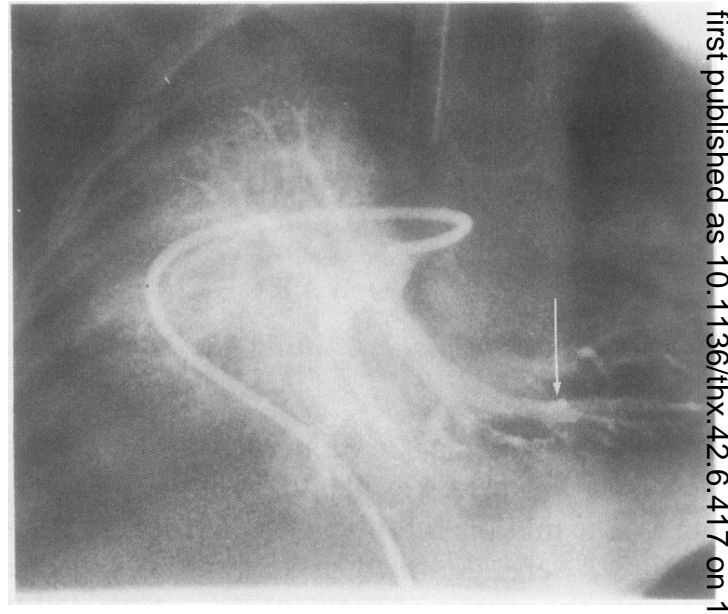

Fig 2 Case 2: Contrast medium selectively injected into the hypoplastic right pulmonary artery showing a branch extending back across the midline into the left hemithorax. This would support the presence of a crossover segment. Although the presence of a right sided pneumothorax is suggested, this was not found; this misleading appearance ha: been noted previously in the scimitar syndrome with hypoplasia of the right lung.

hemithorax (fig 2). This picture was very similar t\$ that found in patient 1 , and strongly suggests thet presence of a crossover segment.

\section{CASE 3}

This girl was referred at the age of 4 months with recurrent chest infections associated with dextro을 cardia. Normal cilial function excluded the diagnosis of Kartagener's syndrome and her symptoms responded well to antibiotics and physiotherapy. He right lung was noted to be hypoplastic and a digita enhanced radiographic study showed a large anoma lous vessel in the right lung, probably a scimitar vein? Clinically she remained well controlled with medicat treatment but persistent changes were still noted oo the chest radiograph at the age of 3 years. A bronv chogram done at this stage showed a rather narrow left bronchus but an otherwise normal left bronchian tree. The right lung was hypoplastic and the bronchiat branching pattern was grossly abnormal (fig 3). Th区 first branch, arising from the site of origin of the nore mal right upper lobe branch, descended medially and crossed the midline, with a segment of the right bron? chial tree outlined in the posteroinferior aspect of the left hemithorax.

\section{Discussion}

These patients show the radiographic features of crossover segment of a lung, found in associatiog 


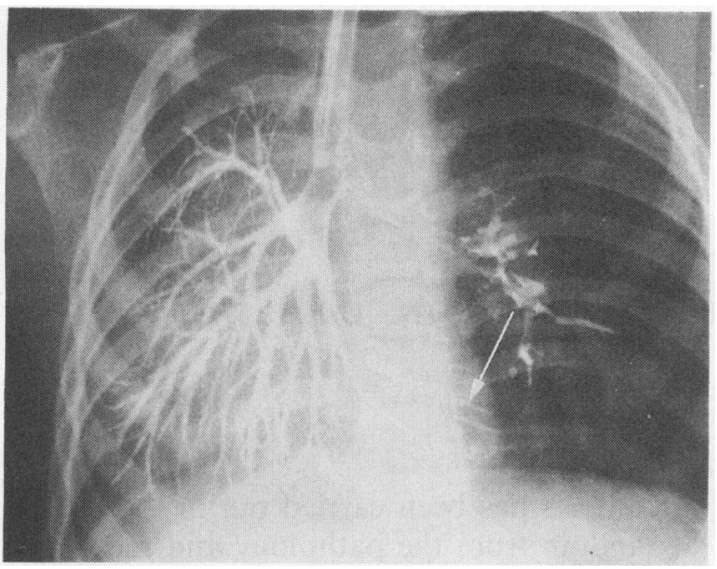

Fig 3 Case 3: Bronchogram showing narrowing of the left main bronchus; the right lung is hypoplastic and the bronchial branching pattern grossly abnormal. A segment of the right bronchial tree arising from the site of the normal upper lobe bronchus is seen crossing the midline into the left hemithorax inferiorly and posteriorly (indicated by arrow).

with a variant of the scimitar syndrome. In case 1 the anatomy was confirmed at thoracotomy. A survey of the relevant publications revealed no reports of such an anomaly. In a recent excellent review of the scimitar syndrome, ${ }^{1}$ however, one of the figures (fig 2) shows a pulmonary arteriogram with a branch from the hypoplastic right pulmonary artery clearly crossing the midline into the left hemithorax. These appearances strongly suggest the presence of a crossover segment.

It is difficult to explain how a segment of hypoplastic lung should come to extend across the midline into the opposite hemithorax. The association with a variant of the scimitar syndrome suggests that all the abnormalities occurred at a similar time early in pulmonary development. The clinical significance of this anomaly is not clear, but its functional effect is probably less important than that attributed to the associated anomalies. Bronchial drainage is likely, however, to be impaired, predisposing this area to recurrent infections. This may have played a part in the complications suffered by the first patient.

We would like to thank Professor Bryan Corrin for his helpful criticism and advice in the preparation of this report.

\section{Reference}

1 Haworth SG, Sauer U, Buhlmeyer K. Pulmonary hypertension in scimitar syndrome in infancy. Br Heart $J$ 1983;50:182-9. 